\title{
Flow Simulation and Identification of Important Model Parameters in Industrial Packed Beds for High-Performance Random Packings
}

\author{
Tatyana Petrova ${ }^{1 *}$, Daniela Dzhonova-Atanasova ${ }^{1}$ \\ 1 Laboratory of transfer processes in multiphase media, Institute of Chemical Engineering, Bulgarian Academy \\ of Sciences, Acad. G. Bonchev Str., Bl. 103, 1113, Sofia, Bulgaria, \\ * Corresponding author's e-mail: t.petrova@iche.bas.bg
}

\begin{abstract}
The goals of this work were: first, to simulate the liquid flow distribution in a large diameter $(1.2 \mathrm{~m})$ packed column with an RMSR 70-5 high performance random packing (layer height up to $3 \mathrm{~m}$ ), by a dispersion model. Second, to find and estimate the important model parameters and flow maldistribution factor, using experimental data and two different optimization approaches. A three-parameter dispersion model for prediction of radial liquid distribution and two different approaches to determine some of the model parameters from experimental data were used. In parallel, a two-parameter optimization procedure for model parameters identification was performed based on the minimum of residual variance between model and experimental liquid velocities over a column crosssection. The simulated and experimental flow maldistribution, were estimated by means of an integral estimation - a maldistribution factor. The comparison between the model and experimental liquid distribution and respective maldistribution factors at packing heights $\mathrm{H}=1 \mathrm{~m}$ and $\mathrm{H}=2.5 \mathrm{~m}$ for liquid load $16.6 \cdot 10^{-3} \mathrm{~m}^{3} / \mathrm{m}^{2} \cdot \mathrm{s}$ showed very good agreement, even for a high packing layer. In conclusion, the presented model predictions and estimations about RSRM 70-packing 5 characteristics and behaviour will complement the information about its efficiency and operation in industrial processes.
\end{abstract}

Keywords: simulation, high-performance packing, optimization, maldistribution factor, RMSR 70-5

\section{INTRODUCTION}

High-performance random packings, as a packed column internals, are widely used for separation processes such as rectification and absorption in chemical industry and environmental protection due to their high efficiency at low pressure drop [5]. The recent interest is connected with the technologies for flue gas scrubbing, heat recovery and fuel production. The uniform liquid distribution in the packed bed cross-section is crucial for the efficiency of the mass transfer processes and for their impact on the environment.

In recent years, there has been an increasing interest in the liquid phase distribution and wall flow observations in larger, industrial columns dimensions [1, 3-4], as well as in the packings of open structure (fourth generation) [7, 9, 11]. These lattice-type open-structure packings are characterized by a complex shape (a kind of a lattice of curved thin lamellae), low pressure drop, and their liquid spreading capacity in the radial direction is weaker than that of conventional packings of previous generations. The latter is due to the absence of liquid discharge surface (s) in the packing element; in practice, the liquid, passing through the packing layer, forms much drops and streams, which is also confirmed by the higher effective surface of these packings than the specific one [5]. For the open-structured packings a distance of 5 to 6 column diameters from the top of the column is required before the "steady conditions" between flow in the wall zone and flow in the bulk zone is attained. It has also been found that the flow in the open-structure packings is very sensitive to the initial distribution.

In the last 10 years, there has only been a limited number of studies about the $4^{\text {th }}$ generation random packings except our research in this field $[3-4,8-9,11]$, which investigate experimentally 
and model the radial liquid distribution and maldistribution [3-4, 9] in those packings. Moreover, there is insufficient information about the radial spreading ability and wall flow effects in these packings $[2,7]$, especially in the column diameters greater than $0.5 \mathrm{~m}$. In contrast, the older type of packings are widely examined in the literature. Therefore, the motivation of our work is to fill the gap and test our models in industrial scale area, based on the existing experimental data for highperformance random packings.

The aim of this study is to simulate and compare with experimental data the liquid distribution in large diameter columns (close to industrial scale) with high performance open-structure random packings. Here, a three-parameter dispersion model for simulation of radial liquid distribution and two different approaches were employed to determine some of the model parameters from the experimental data. In parallel, two-parameter optimization procedure for model parameters identification was performed based on the minimum of residual variance between model and experimental liquid velocities over a column cross-section. The simulated and experimental flow maldistribution was estimated by an integral estimation - a maldistribution factor. The experimental data for liquid distribution in an RMSR 70-5 high-performance random packing for a packed column with a diameter of $1.2 \mathrm{~m}$ and packing layer heights up to $3 \mathrm{~m}$, for $16.6 \cdot 10^{-3} \mathrm{~m}^{3} / \mathrm{m}^{2} \cdot \mathrm{s}$ liquid load and two types of initial irrigation (uniform and point source), were kindly provided by Florian Hanusch from the University of Munich, Germany.

By the two above-mentioned methods, two of the model parameters were estimated, using the experimental data for the wall flow at different packing heights at initial uniform irrigation, and also the data for point source initial irrigation, measured in a $1.2 \mathrm{~m}$ diameter column RMSR 70-5 for random packing. Finally, the comparison between model and experimental liquid distribution and maldistribution factors at packing heights $\mathrm{H}$ $=1 \mathrm{~m}$ and $\mathrm{H}=2.5 \mathrm{~m}$ for liquid load $16.6 \cdot 10^{-3}$ $\mathrm{m}^{3} / \mathrm{m}^{2} \cdot \mathrm{s}$, shows very good agreement, even for a higher packing layer.

\section{MATERIALS AND METHODS}

The simulation was performed using a dispersion model created [10] and widely used in recent years for predicting the liquid distribution for older packing generations and for small diameter columns. It was tested successfully in the near past for a $0.5 \mathrm{~m}$ column diameter and Raschig Super-Rings [7], and for Pall rings and a column diameter of $0.6 \mathrm{~m} \mathrm{[6]}$. Here, for brevity, only the dispersion model solutions for uniform initial irrigation and the physical meaning of the model parameters were presented.

Analytical dimensionless solutions for the irrigation density $f^{u}$ and for the wall flow $W^{u}$ of the above-mentioned model in the form of infinite series at uniform initial irrigation are given below:

$$
\begin{aligned}
& f^{u}(r, z)=A_{0}+\sum_{n=1}^{\infty} A_{n}^{u} J_{0}\left(q_{n} r\right) \exp \left(-q_{n}^{2} z\right), \\
& W^{u}=\frac{1}{1+C}-2 \sum_{n=1}^{\infty} A_{n}^{u}\left(q_{n}\right) \frac{J_{1}\left(q_{n}\right)}{q_{n}} \exp \left(-q_{n}^{2} z\right)
\end{aligned}
$$

with coefficients:

$$
A_{0}=\frac{C}{1+C}, \dot{A}_{n}^{u}=\frac{2\left(q_{n}^{2} / B-2 C\right)}{\left[\left(q_{n}^{2} / B-2 C\right)^{2}+q_{n}^{2}+4 C\right] J_{0}\left(q_{n}\right)}
$$

The parameter $B$ is a criterion for exchange of liquid between the column wall and the packing; parameter $C$ expresses the equilibrium distribution of entire liquid flow between the wall and the packing when equilibrium state is attained $z \rightarrow \infty$. In Eq. (1) and (2) $J_{0}, J_{1}$ are Bessel functions of the first kind, zero and first order; $q_{n}$ are the roots of the characteristic equation, following from boundary condition at the column wall. In the dimensionless axial coordinate $z=$ $D \cdot H / R^{2}$ the third model parameter is included $D$ is the packing radial liquid distribution coefficient, [m], also called packing spreading coefficient. $\mathrm{H}$ is the packing layer height [m], and $\mathrm{R}$ is the column radius [m].

As explained above, three model parameters should be determined $-C, D$ and $B$. In the light of the experimental data used in this paper, $C$ is calculated from the wall flow data for $\mathrm{L}_{0}=60$ $\left[\mathrm{m}^{3} / \mathrm{m}^{2} \cdot \mathrm{h}\right], \mathrm{G}=0\left(\mathrm{G}\right.$ is the gas load $\left.\left[\mathrm{m}^{3} / \mathrm{m}^{2} \mathrm{~h}\right]\right)$, collected for uniform initial irrigation for packing RMSR 70-5 at heights of $1 \mathrm{~m}, 1.5 \mathrm{~m}, 2.5 \mathrm{~m}$ and 3 m. In [6] the relation $C=1-W_{z \rightarrow \infty} / W_{z \rightarrow \infty}$ is developed, which is practically the case, when in Eq. (1) for model wall flow the second term disappears at $z \rightarrow \infty$. The calculated value is $C=5.944$.

The other two parameters $-B, D$ can be identified by non-linear optimization approach, minimizing the residual variance $S_{A}^{2}$ : 


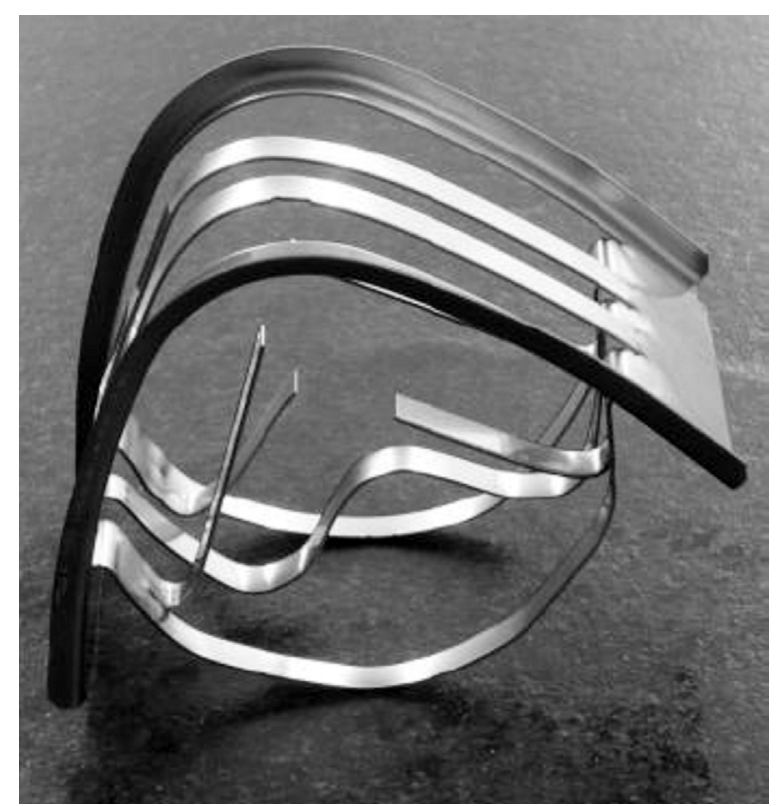

Fig. 1. Photograph of high-performance random packings used in this study RMSR, RVT Process Equipment

$$
S_{A}^{2}=\frac{1}{k-1} \sum_{i=1}^{k} n_{i}\left(f_{\dot{i}}-f_{\dot{c}}\right)^{2}
$$

where $n_{i}$ is the number of packing redumpings,

$f_{i}$ is the mean dimensionless density of irrigation (flow velocity) in $i$-th annular section of the $7^{\text {th }}$ segment liquid collecting device, delimited by the radii $r_{i-1}$ and $r_{i}\left(r_{i}>r_{i-1}\right)$ and it is determined by the expression:

$$
f_{i}=\frac{2}{r_{i}^{2}-r_{i-1}^{2}} \int_{r_{i-1}}^{r_{i}} f(r, z) r d r
$$

Here the experimental and theoretically calculated values with "e" and "c" indices (Eq. (1)) of the liquid density of irrigation are denoted. If the global minimum of $S_{A}^{2}$ exists for some optimal values of $B$ and $D$, searching in physically possible intervals for these two parameters, then they can be identified. As it was found in [7], in the case $S_{A}^{2}$ of invariance in respect to parameter $D$, this global minimum cannot be found and the other approaches have to be used.

In the case of unknown value of $D$ for the packing considered, another approach (single jet method) is developed, which is explained in detail in [2], and needs experimental data for radial liquid distribution, measured after relatively short packing layer and at central point initial irrigation. Fortunately, such data was available and we applied the single jet method to determine $D$ for a packing RMSR 70-5. The results are presented in Figure 2. For older packing types, usually $D$ depends on the liquid initial density (velocity) of irrigation. Here, the same situation can be observed - the values for $\mathrm{L}=10 \mathrm{~m}^{3} / \mathrm{m}^{2} \cdot \mathrm{h}$ and $\mathrm{L}=$ $15 \mathrm{~m}^{3} / \mathrm{m}^{2} \cdot \mathrm{h}$ practically coincide. The determined mean value of $D$ by the single jet method [7] for packing RMSR 70-5 is $D=0.009062 \mathrm{~m}$, using Eq. (5), where $q=L / Q,\left[m^{2}\right]$ :

$$
\ln (q)=\ln \left(\frac{1}{4 \pi H D}\right)-\frac{r^{2}}{4 D H}
$$

As mentioned above, we can also try the twoparameter identification procedure to determine $B, D$ for the RMSR 70-5 packing by searching the minimal value of $S_{A}^{2}$, using the Eq. (1) and (4)

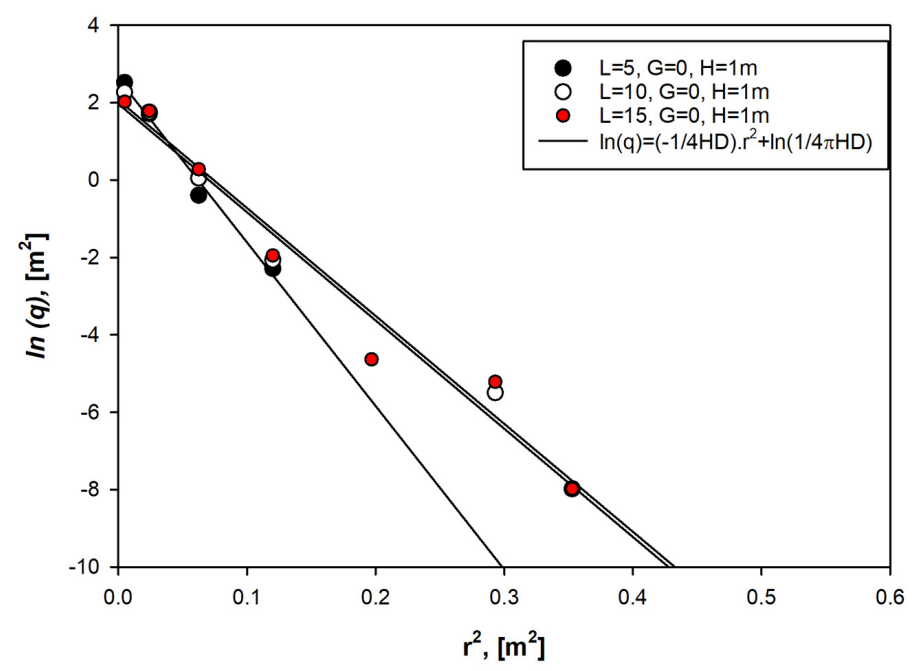

Fig. 2. Values of $\ln (q)$ versus $r^{2}$ according to Eq.(5) for determination of radial spreading coefficient for packing RMSR 70-5 with single jet method 

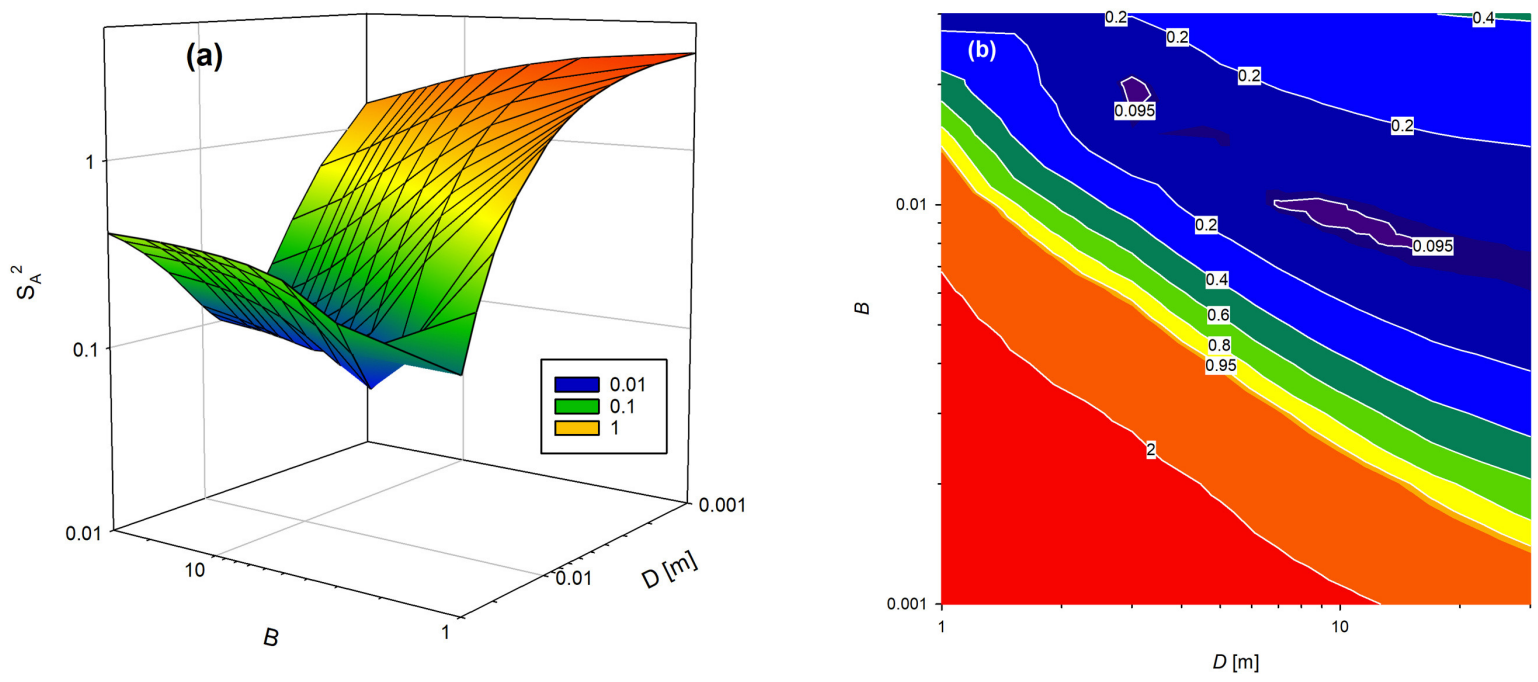

Fig. 3. Two -parameter identification of model parameters with optimum criteria - minimal value of residual variance $=0.91 \mathrm{e}-01$ at $D=0.0095 \mathrm{~m}, B=10, C=5.944$, for $\mathrm{H}=1 \mathrm{~m}$, packing RMSR 70-5 presented as 3D plot (a), and presented as contour plot (b)

for model mean dimensionless density of irrigation $f_{i c}$, and experimental data for radial distribution of $f_{i e}$. The results of the two-parameter identification were successfully obtained and they are illustrated in Figure 3 (a) and (b). The values of $D$ from the single-jet method and from the twoparameter identification procedure (although different experimental data are used), are very close $-0.00906 \mathrm{~m}$ and $0.0095 \mathrm{~m}$, which confirms the results in general for the considered packing.

\section{RESULTS AND DISCUSSION}

With the found values of the model parameters, i.e, $D=0.0095 \mathrm{~m}, B=10, C=5.944$, the

(a)

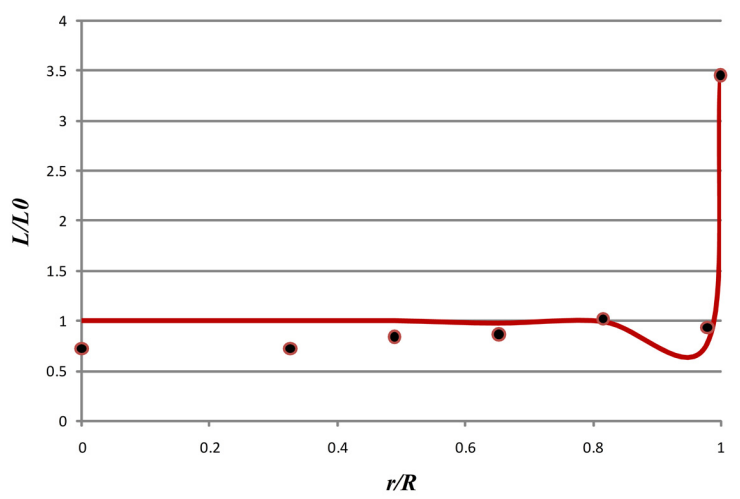

model solution was calculated by Eq. (1) at packing heights $\mathrm{H}=1 \mathrm{~m}$ and $\mathrm{H}=2.5 \mathrm{~m}$ and compared with respective experimental data for initial uniform irrigation, for initial liquid load $\mathrm{L}_{0}=60$ $\mathrm{m}^{3} / \mathrm{m}^{2} \cdot \mathrm{h}$, or $16.6 \cdot 10^{-3} \mathrm{~m}^{3} / \mathrm{m}^{2} \cdot \mathrm{s}$. The comparison between the model and experimental liquid distribution shows very good agreement, even for a higher packing layer (Figure 4 (a) and (b)).

The model and experimental radial liquid distribution results can also be estimated by means of the maldistribution factors. The formula for calculating the maldistribution factor is given in [3] as:

$$
M_{f}=\frac{1}{F_{0}} \sum_{i=1}^{N} F_{i}\left|\frac{L_{i}-L_{0}}{L_{0}}\right|
$$

(b)

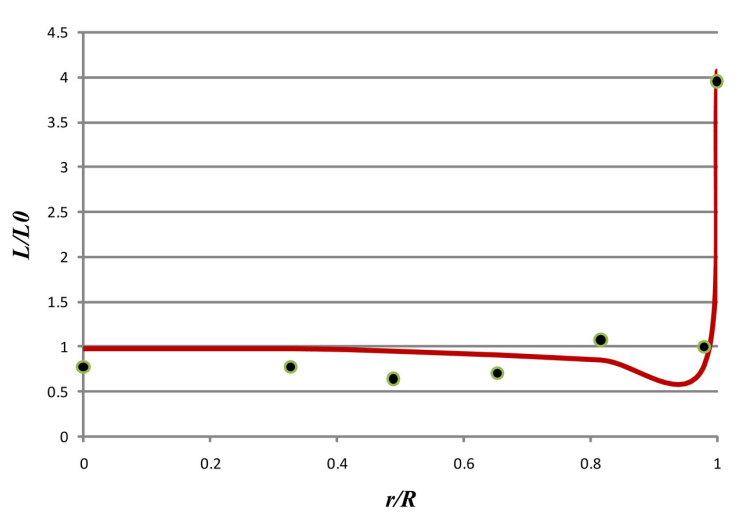

Fig. 4. Comparison between liquid radial distributions obtained by dispersion model in this work and experimental data for RMSR 70-5, $\mathrm{H}=1 \mathrm{~m}, \mathrm{~L}=16.6 \cdot 10^{-3} \mathrm{~m}^{3} / \mathrm{m}^{2} \cdot \mathrm{s}$, $\mathrm{G}=0$ (a), and for RMSR 70-5, $\mathrm{H}=2.5 \mathrm{~m}, \mathrm{~L}=16.6 \cdot 10^{-3} \mathrm{~m}^{3} / \mathrm{m}^{2} \cdot \mathrm{s}, \mathrm{G}=0$ (b) 
In Eq. (6) the ratio $F_{i} / F_{0}$ represents the ratio of the area of the respective segment $i$ to the entire area of the column section, $\mathrm{m}^{2}, n s$ is the number of segments of the liquid collecting device. Accordingly, $L_{i} / L_{0}$ is the mean dimensionless theoretical or experimental irrigation density in the $i$ segment, delimited by the $r_{i-1}$ and $r_{i}\left(r_{i}>r_{i-1}\right)$ radii, which is obtained from the dispersion model according to Eq. (4). As can be seen from Eq. (1) and (6) the model maldistribution factor and the solution depends on $r, z$ and the $B, C$ and $D$ dispersion model parameters for the respective packing and current packing layer height $z=D \cdot H / R^{2}$. Theoretical $M_{f}$, calculated for optimal parameter values are $M_{f}=0.172$ for $\mathrm{H}=1 \mathrm{~m}$, and $M_{f}=0.242$ for $\mathrm{H}=2.5$ $\mathrm{m}$. The corresponding experimental ones are $M_{f}=$ 0.198 (for $\mathrm{H}=1 \mathrm{~m}$ ) and $M_{f}=0.265$ for $\mathrm{H}=2.5 \mathrm{~m}$. This again confirms that the model predictions are in good agreement with the experimental data.

\section{CONCLUSIONS}

The obtained results confirm the dispersion model ability to successfully simulate the radial liquid flow distribution in a packed column of a large diameter of $1.2 \mathrm{~m}$ with a RMSR 70-5 highperformance random packing. Two different approaches (two-parameter identification and single jet method) are independently applied to obtain one of the model parameters - the coefficient of radial spreading of the RMSR 70-5 packing. The values of $D$ are very close. The other two model parameters were also determined and the comparison between model results for the liquid distribution and respective experimental data at two packing heights shows good agreement. The model and experimental maldistribution factors of the liquid distribution also are very close to each other. The presented model predictions and estimations about the RSRM 70-5 packing characteristics and behaviour will complement the information about its efficiency and operation in industrial processes.

\section{Acknowledgements}

This work was financially supported by the National Science Fund at the Bulgarian Ministry of Education and Science, Contract No DN 07/14/ 15.12.2016. The authors also express their special thanks to Florian Hanusch from the University of Munich, Germany for the access to the experimental data, obtained during project "Cell Model for Design of Packed Columns and Liquid Distributors", (http:// www.ext.apt.mw.tum.de/maldistribution_en/data).

\section{REFERENCES}

1. Dzhonova-Atanasova D., Petrova T., Semkov Kr., Darakchiev S., Stefanova K., Nakov S., Popov R. 2018. Experimental investigation of liquid distribution in open structure random packings as a basis for model refinement. Chemical Engineering Transactions, 70, 2077-2082.

2. Dzhonova-Atanasova D., Kolev N., Nakov Sv. 2007. Determination of liquid radial spreading coefficients of some highly effective packings. Chemical engineering Technology, 30 (2), 202-207.

3. Hanusch F., Rehfeldt S., Klein H. 2018a. Liquid maldistribution in random packed columns: experimental investigation of influencing factors. Chemical Engineering and Technology, 41(11), 2241-2249.

4. Hanusch F., Engel V., Kender R., Rehfeldt S., Klein H. 2018b. Development and application of the TUM-WelChem cell model for prediction of liquid distribution in random packed columns. Chemical Engineering Transactions, 69, 739 -744.

5. Hegely L., Roesler J., Alix P., Rouzineau D., Meyer M. 2017. Absorption methods for the determination of mass transfer parameters of packing internals: A literature review. AIChE Journal, 63(8), 3246-3275.

6. Petrova T., Stefanova K., Dzhonova-Atanasova D., Semkov Kr. 2017. Modeling of a liquid spreading in randomly packed metal Pall rings. Proc. Int. Sci. Conf. MATHMODEL 2017, 26-29.

7. Petrova T., Semkov Kr., Dzhonova-Atanasova D. 2018. Modeling of liquid distribution in a packed column with open-structure random packings. Chemical Engineering Transactions, 70, 1051-1056.

8. Schug S. 2018. Untersuchung der Fluiddynamik in Packungskolonnen mittels Computertomographie. Ph.D.Thesis, Der Technischen Fakultät der Friedrich-Alexander-Universität Erlangen-Nürnberg, Erlangen-Nürnberg, Germany (in German).

9. Schultes M., Brauer J., Chen P., Doong S. 2018. Marinization of mass transfer columns for FLNG applications. Chemical Engineering Transactions, 69, 301-306.

10. Staněk V., Kolář V. 1968. Distribution of liquid over a random packing: Verification of the boundary condition of liquid transfer between a packed bed and the wall of a cylindrical column and evaluation of its parameters. Collection of Czechoslovak Chemical Communications, 33(4), 1062-1077.

11. Van Holt F., Brinkmann J., Grunewald M.2018.Experimental and theoretical studies on local liquid phase distribution in packed columns. Chemical Engineering Transactions, 69, 391-396. 